\title{
Expression pattern of aquaporins in patients with primary nephrotic syndrome with edema
}

\author{
YU WANG ${ }^{*}$, JIMEI BU* ${ }^{*}$ QING ZHANG, KAI CHEN, JIHONG ZHANG and XIAORONG BAO \\ Department of Nephrology, Jinshan Hospital Affiliated to Fudan University, Shanghai 201508, P.R. China
}

Received July 15, 2014; Accepted April 20, 2015

DOI: $10.3892 / \mathrm{mmr} .2015 .4209$

\begin{abstract}
The association between the expression of aquaporins (AQPs) in kidney tissues and the occurrence of edema in nephrotic syndrome (NS) remains unclear. The current study aimed to investigate this association. A total of 54 patients with primary glomerular disease, diagnosed by renal biopsy, were divided into three groups: Control, NS without edema and NS with edema. The expression of AQP1, AQP2, AQP3 and AQP4 in kidney tissues from these patients was assessed using immunohistochemistry, and urinary AQP concentrations were quantified by ELISA. Comparison of the three groups was conducted using one way analysis of variance, independent samples t-test or the Chi-square test. AQP1 was strongly expressed in the proximal tubules. The proportion of the AQP1-positive area in kidney tissues from patients with NS with edema was significantly reduced, in comparison with the other two groups. By contrast, the proportion of the AQP2-positive area in the NS with edema group was significantly higher than that of the other two groups; significant differences were also observed between the control and NS without edema groups for this parameter. Urinary AQP2 concentrations in patients with NS (with and without edema) were significantly higher than that of the control group, and exhibited a significant positive correlation with kidney tissue AQP2 concentrations. The present study demonstrated the abnormal expression pattern of AQP1-AQP4 in the kidney tissues of patients with NS, providing a basis for an improved understanding of the role of AQP in the pathogenesis of NS.
\end{abstract}

\section{Introduction}

Nephrotic syndrome (NS), characterized by edema, proteinuria, hypoalbuminemia and hyperlipemia, is nonspecific form of kidney disease. NS occurs as a result of any disorder that damages the nephrons, including diabetes, vasculitis, lupus and

Correspondence to: $\mathrm{Dr}$ Xiaorong Bao, Department of Nephrology, Jinshan Hospital Affiliated to Fudan University, 1508 Longhang Road, Shanghai 201508, P.R. China

E-mail: xiaorongbaomedsci@163.com

Key words: aquaporin, nephrotic syndrome, edema nephritis. NS affects the integrity of the glomerular capillary membrane and results in an inflammatory response in the glomeruli (1). It may occur in any age group, although it is most prevalent in children between 18 months and 4 years of age. The incidence is greater in boys than girls, although there is an approximately equal sex distribution in adults (2). The most common manifestation of NS is edema, which is considered to be a result of the reduced plasma colloid osmotic pressure that results from proteinuria and hypoproteinemia. However, clinical observations have suggested otherwise, abnegating the association between edema and hypoproteinemia in patients with NS, whose serum albumin levels may be normal or low (3).

Aquaporins (AQPs) are low molecular-weight hydrophobic and transmembrane proteins, the monomers of which range between 26 and $34 \mathrm{kDa}$ in size. AQPs facilitate a diffusion rate that is $10-20$ times faster than simple diffusion, and thus their selective permeability to water is very high (4). To date, 13 AQP subtypes, AQP0-AQP12, have been described (5). AQPs exhibit similar water-permeating functions, but serve different physiological roles and are expressed in different tissue areas (6). AQPs are widely distributed in body tissues and cells, particularly in epithelial cells that are associated with fluid secretion and absorption (5). The kidney, one of the primary regulators of water and salt metabolism, expresses numerous subtypes of AQPs, including AQP1, AQP2, AQP3, AQP4, AQP6, AQP7 and AQP8, which are predominantly localized in the proximal tubules, collecting ducts and thin limbs of the loop of Henle, and participate in the regulation of water transportation in the kidney (7-9). Previous studies have suggested an association between abnormal AQP expression and edema-associated diseases, such as congestive cardiac failure and hepatic cirrhosis (10-13). However, the association between the expression of AQPs in kidney tissues and edema in NS remains unclear.

Due to the fact that AQP1-4 are the primary types of AQPs in the kidney, the association between AQP1-4 expression and edema was investigated in kidney tissues from patients with primary NS in the current study.

\section{Materials and methods}

The current study was approved by the Institutional Review Board of the Jinshan Hospital Affiliated to Fudan University (Shanghai, China) and written informed consent was obtained from each participant. 
Cases and groups. Patients admitted to the hospital and diagnosed with primary glomerular disease [including immunoglobulin A ( $\operatorname{Ig} \mathrm{A})$ nephropathy] by renal biopsy, were eligible for the study. Inclusion criteria were as follows: Patients were $>18$ years old; diuretics, corticosteroids and renin-angiotensin-aldosterone system blockers were not administered prior to biopsy; no other diseases, such as hepatic cirrhosis and cardiac dysfunction, which may result in edema, were present; no electrolyte imbalance was detected; patients had provided informed consent.

Patients were divided into NS and control groups, according to the following diagnostic criteria for NS: Macroalbuminuria ( $\geq 3.5 \mathrm{~g} /$ day) and hypoproteinemia (<30 g/l). Patients with NS were further subdivided into NS without edema and NS with edema groups, based on the presence or absence of pitting edema (for example of the eyelids, dorsal and anterior tibial muscles and sacrum).

Materials. The EliVision Plus kit and 3,3'-diaminobenzidine (DAB) kit were obtained from Fuzhou Maixin Biotechnical Co., Ltd. (Fuzhou, China). DAB-1031 and hematoxylin staining solution were obtained from Nanjing Jiancheng Bioengineering Institute (Nanjing, China). Neutral gum was obtained from Sinopharm Chemical Reagent Co., Ltd (Shanghai, China). Polylysine solution, phosphate-buffered saline (PBS) and sodium citrate buffer were obtained from Nanjing KeyGen Biotech. Co., Ltd. (Nanjing, China).

Biochemical assays. Blood urea nitrogen (BUN), serum creatinine (Scr), albumin, potassium and sodium were detected using a Beckman CX9 Biochemical Analyzer (Beckman Coulter, Brea, CA, USA) and Beckman designated reagents. Urine protein (24-h) was quantified on a Johnson Vitros-350 Biochemical Analyzer (Johnson \& Johnson, Shanghai, China).

Glomerular filtration rate (GFR) calculation. The Modification of Diet in Renal Disease equation: eGFR $=170 \times$ Scr $^{-0.999} \mathrm{x}$ Age $^{-0.176} \times$ BUN $^{-0.170} \times$ Alb $^{0.318} \times(0.762$ Female $)$, was applied to assess the estimated GFR (eGFR).

Processing of kidney tissues. A portion of renal tissues, obtained from kidney biopsies, were pathologically examined by the Research Institute of Nephrology of Fudan University (Shanghai, China). The remaining tissue samples were preserved in $70 \%$ ethanol (Shanghai Jiuyi Chemical Reagent Co., Ltd., Shanghai, China) at $4^{\circ} \mathrm{C}$ in a refrigerator, following fixation with $4 \%$ formaldehyde for $24 \mathrm{~h}$.

Immunohistochemistry. Naturally dried tissue samples were immersed in 4\% paraformaldehyde (Shanghai Jiuyi Chemical Reagent Co., Ltd.) solution for $30 \mathrm{~min}$ in order to improve cell permeability. Following washing three times in PBS for 3 min each time, samples were successively dewaxed by incubation with xylene (Shanghai Jiuyi Chemical Reagent Co., Ltd.) and rehydrated with an ethanol gradient (successively anhydrous; 95 , 85 and $70 \%$ ) for $5 \mathrm{~min}$ at each step. The slides were placed into the antigen retrieval buffer $(3 \mathrm{~g}$ trisodium citrate, $0.4 \mathrm{~g}$ citric acid, $1 \mathrm{~L}$ distilled water; Shanghai Jiuyi Chemical Reagent Co., Ltd.) and boiled for $20 \mathrm{~min}$. Following 3 washes with PBS, 2 drops of $3 \% \mathrm{H}_{2} \mathrm{O}_{2}$-methanol (800 $\mu \mathrm{l}$ methanol, $100 \mu \mathrm{l}$ water, $100 \mu 1 \mathrm{H}_{2} \mathrm{O}_{2}$;
Shanghai Jiuyi Chemical Reagent Co., Ltd.) were added to each slide for endogenous peroxidase inhibition. Subsequently, the slides were incubated with 50-100 $\mu$ l normal goat serum (Wuhan Boster Biological Technology Ltd., Wuhan, China) for $10 \mathrm{~min}$ at room temperature. Samples were then successively incubated with primary rabbit polyclonal antibodies targeting AQP1 (cat. no. BA0648), AQP2 (cat. no. BA0649), AQP3 (cat. no. BA1559) and AQP4 (cat. no. (BA1560) (1:200 dilutions; Wuhan Boster Biological Technology Ltd.) in a humid chamber for $2 \mathrm{~h}$, and an enhancer (Fuzhou Maixin Biotechnical Co., Ltd.) for $30 \mathrm{~min}$ at room temperature). Following 3 washes with PBS, the slides were incubated at $37^{\circ} \mathrm{C}$ for $30 \mathrm{~min}$ with goat anti-rabbit secondary antibody conjugated to horseradish peroxidase (cat. no. KIT-9902; Fuzhou Maixin Biotechnical Co., Ltd.). For detection, 2 drops of freshly prepared DAB solution was applied to each slide. The color reaction was terminated with a 15-min wash in distilled water and slides were counter-stained with hematoxylin for $10 \mathrm{~min}$. Tissue samples were successively incubated with 70, 85, 95\% and anhydrous ethanol (5 min each) and then twice with xylene (10 min each step) at $37^{\circ} \mathrm{C}$. Finally, neutral gum and coverslips were successively applied following natural drying of the slides. The expression of each AQP subtype was assessed by a blinded individual using an optical microscope (BX43; Olympus Corporation, Tokyo, Japan), and micrographs of three areas were captured for each slide. The positive index was calculated as a ratio of immunoreactive area to the total tissue area. The positive index of each slide was the average obtained from three fields.

Examination of urine samples. Urine specimens were collected over $24 \mathrm{~h}$ ( 6 am to 6 am the next day) in a container, and measured in a dosage cup. The total protein concentrations were then measured using a Johnson Vitros-350 Biochemical Analyzer and AQP concentrations were determined by ELISA. Finally, 24-h total urine protein and AQP quantities were deduced.

Data processing and statistical analysis. Data are expressed as the mean \pm standard deviation and statistical analyses were performed using SPSS software, version 13.0 (SPSS, Inc., Chicago, IL, USA). Continuous variables were compared among groups using one way analysis of variance and the independent samples t-test for two groups, while discrete variables were compared using the Chi-square test. $\mathrm{P}<0.05$ was considered to indicate a statistically significant difference. Single correlation analysis was used to characterize the association between variables.

\section{Results}

Clinical data. Among the 54 patients enrolled, 21 who did not have NS, were assigned to the control group, including 1 case of focal segmental glomerulosclerosis (FSGS), 15 individuals with IgA nephropathy and 5 cases of mesangial proliferative glomerulonephritis. The NS without edema group contained 16 patients ( 7 cases of FSGS, 1 case of membranous nephropathy and 8 cases of $\operatorname{Ig}$ A nephropathy). The NS with edema group contained 17 patients ( 3 cases of FSGS, 10 patients with membranous nephropathy, 3 cases of $\operatorname{IgA}$ nephropathy and 1 case of minimal change nephropathy). As presented in 
Table I. Clinical data, urinary protein and serum markers of the three groups.

\begin{tabular}{|c|c|c|c|}
\hline Variable & Control group $(\mathrm{n}=21)$ & NS without edema group $(n=16)$ & NS with edema group $(n=17)$ \\
\hline Age (years) & $41.5 \pm 14.4$ & $42.4 \pm 13.3$ & $42.4 \pm 16.6$ \\
\hline Gender (M/F) & $9 / 12$ & $7 / 9$ & $8 / 9$ \\
\hline GFR (ml/min) & $98.85 \pm 23.27$ & $95.06 \pm 29.24$ & $85.27 \pm 21.43$ \\
\hline Albumin (g/l) & $42.10 \pm 3.86$ & $26.38 \pm 3.14^{\mathrm{a}}$ & $25.59 \pm 3.22^{\mathrm{b}}$ \\
\hline Urinary protein $(\mathrm{g} / 24 \mathrm{~h})$ & $0.56 \pm 0.28$ & $4.57 \pm 1.05^{\mathrm{a}}$ & $5.46 \pm 1.74^{\mathrm{b}}$ \\
\hline Sodium (mmol/l) & $140.33 \pm 3.07$ & $140.44 \pm 3.58$ & $139.06 \pm 2.46$ \\
\hline Potassium (mmol/l) & $3.97 \pm 0.33$ & $4.06 \pm 0.35$ & $4.00 \pm 0.30$ \\
\hline
\end{tabular}

Data are presented as the mean \pm standard deviation. ${ }^{\mathrm{a}} \mathrm{P}<0.01$, compared with the control group, ${ }^{\mathrm{b}} \mathrm{P}<0.01$, compared with the control group. NS, nephrotic syndrome; GFR, glomerular filtration rate.

Table II. Expression of AQPs in kidney tissues.

Positive index $(\%)$

\begin{tabular}{lccc}
\cline { 2 - 3 } AQP & Control group & NS without edema group & NS with edema group \\
\hline 1 & $0.683 \pm 0.311$ & $0.652 \pm 0.300$ & $0.414 \pm 0.201^{\mathrm{b}, \mathrm{c}}$ \\
2 & $0.512 \pm 0.213$ & $0.665 \pm 0.228^{\mathrm{a}}$ & $0.823 \pm 0.002^{\mathrm{b}, \mathrm{c}}$ \\
3 & $0.585 \pm 0.111$ & $0.592 \pm 0.119$ & $0.614 \pm 0.114$ \\
\hline
\end{tabular}

${ }^{\mathrm{a}} \mathrm{P}<0.05$, compared with the control group, ${ }^{\mathrm{b}} \mathrm{P}<0.01$, compared with the control group, ${ }^{\mathrm{c}} \mathrm{P}<0.05$, compared with the NS without edema group. AQPs, aquaporins; NS, nephrotic syndrome.

Table III. Urinary AQP levels.

\begin{tabular}{lccc}
\hline & & Quantity in 24 h urine sample (ng/ml) & \\
\cline { 2 - 4 } AQP & Control group & NS without edema group & NS with edema group \\
\hline 1 & $39.189 \pm 12.448$ & $41.492 \pm 14.766$ & $43.078 \pm 17.923$ \\
2 & $30.320 \pm 9.528$ & $38.621 \pm 13.187^{\text {a }}$ & $45.309 \pm 16.921^{\mathrm{b}}$ \\
\hline
\end{tabular}

Data are presented as the mean \pm standard deviation. ${ }^{\mathrm{a}} \mathrm{P}<0.05$, compared with the control group, ${ }^{\mathrm{b}} \mathrm{P}<0.01$, compared with the control group. AQP, aquaporin; NS, nephrotic syndrome.

Table I, no statistically significant differences were observed in gender and age distributions among the three groups. Serum albumin levels in patients with NS (with or without edema) were significantly lower compared with the control group (both $\mathrm{P}<0.05$ ); while the NS without edema and NS with edema groups exhibited similar albumin levels (both $\mathrm{P}>0.05$ ). Finally, eGFR, potassium and sodium levels were not statistically different among the three groups ( $\mathrm{P}>0.05$; Table I).

AQP1 expression in kidney tissues and urinary AQP1 levels. AQP1 was strongly expressed in the proximal tubules, and was not detected in the glomerulus (Fig. 1). The positive index of AQP1 in samples from patients with NS with edema was significantly lower than that obtained from the control and NS without edema groups $(\mathrm{P}<0.05)$; while the latter two groups yielded similar values $(\mathrm{P}>0.05$; Table II). The quantity of
AQP1 in the urine in the NS with edema group was higher than the that in the other two groups, although these differences were not statistically significant $(\mathrm{P}>0.05$; Table III). Finally, urine AQP1 levels were not statistically associated with AQP1 expression in kidney tissues ( $\mathrm{P}>0.05$; Table IV).

AQP2 expression in kidney tissues and urinary AQP2 levels. AQP2 was strongly expressed in the collecting ducts, and was not detected in the glomerulus (Fig. 2). The AQP2 positive indices in patients with NS (with or without edema) were significantly higher than the index of the control group (both $\mathrm{P}<0.05$; Table II). The quantity of AQP2 in the urine of patients with NS (with or without edema) was significantly higher than that in the control group (both $\mathrm{P}<0.05$ ). However, no statistically significant difference was detected between the two NS groups ( $P>0.05$, Table III). Notably, the quantity 


\section{Control group}

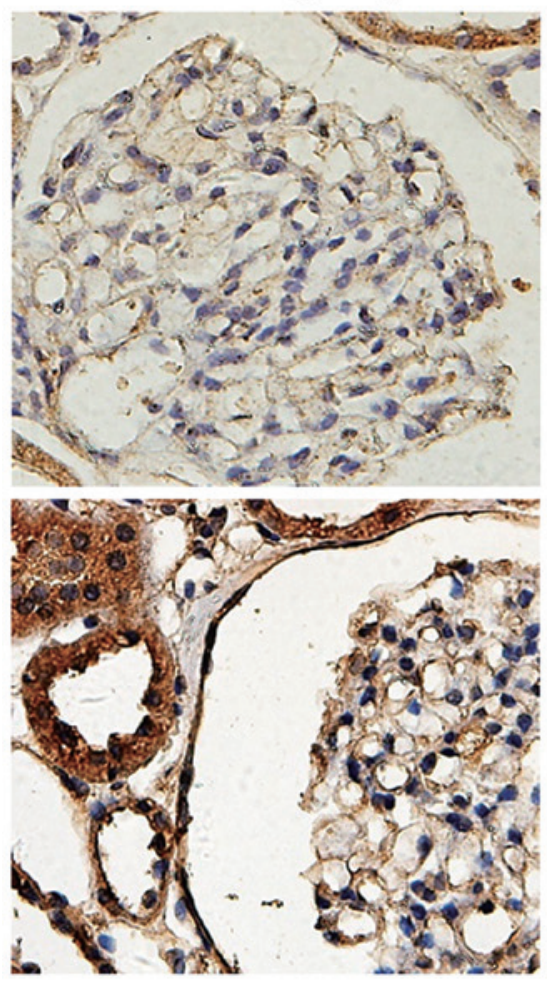

NS without edema
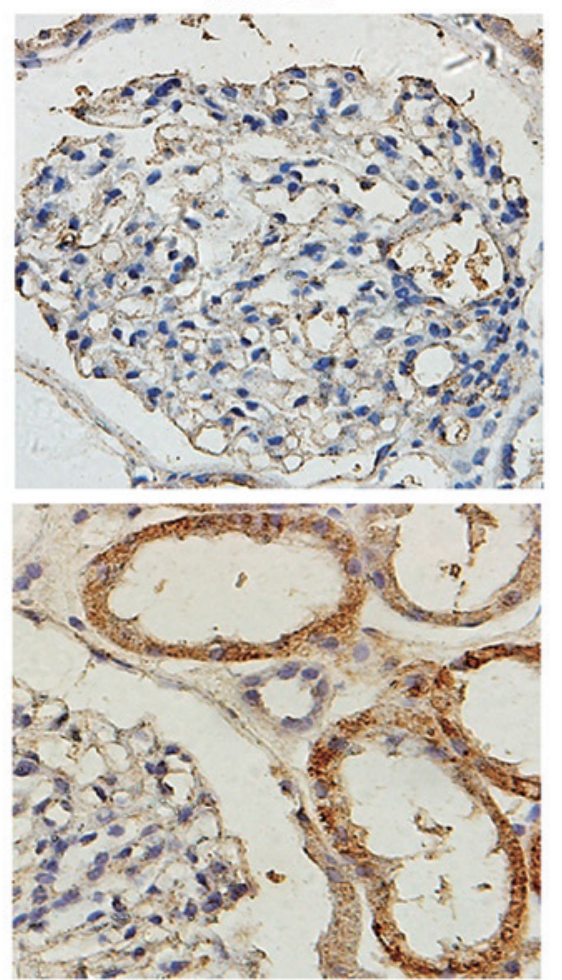

\section{NS with} edema
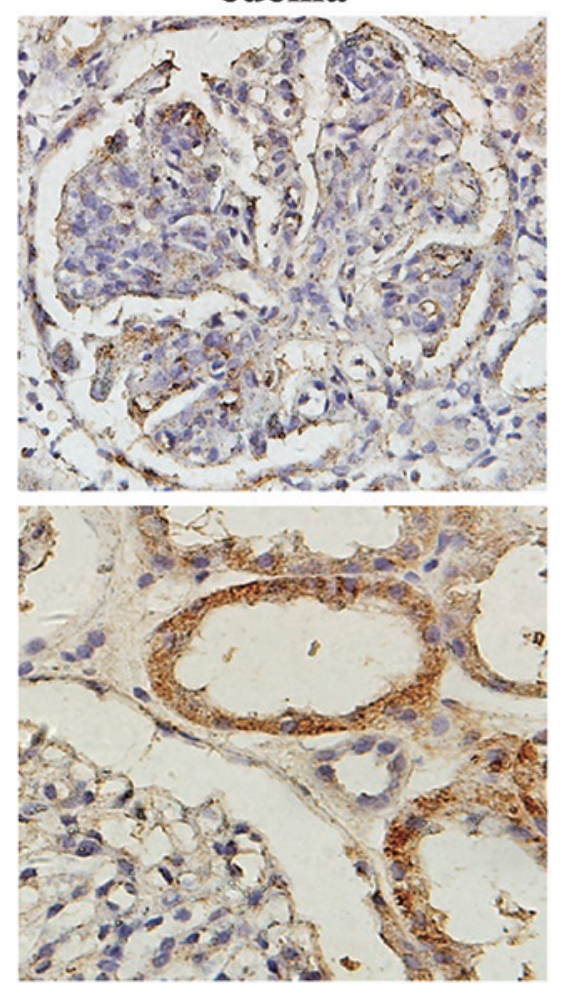

Figure 1. Expression of AQP1 in kidney tissues in the control, NS without edema and NS with edema groups (magnification, $\mathrm{x} 400$ ). Representative micrographs in each group demonstrate the immunostaining results for AQP1 in renal tubules (bottom panel) and glomeruli (top panel). AQP, aquaporin; NS, nephrotic syndrome.

\section{Control group}
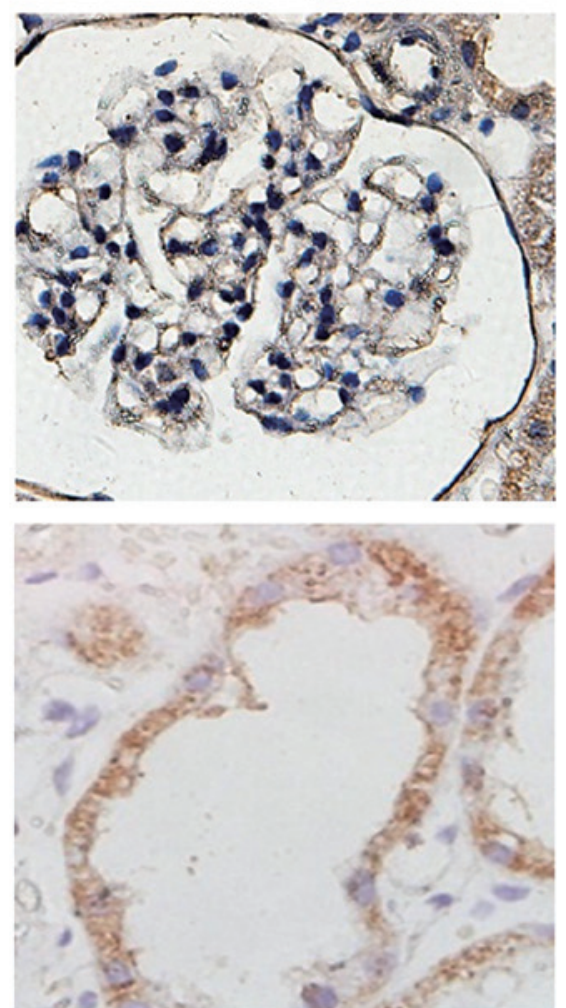

NS without edema
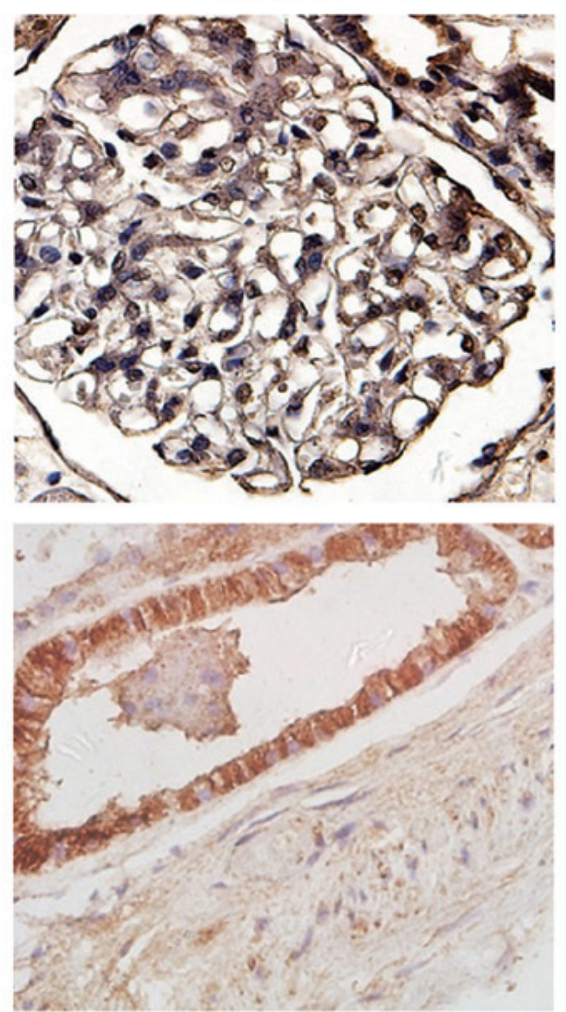

NS with edema
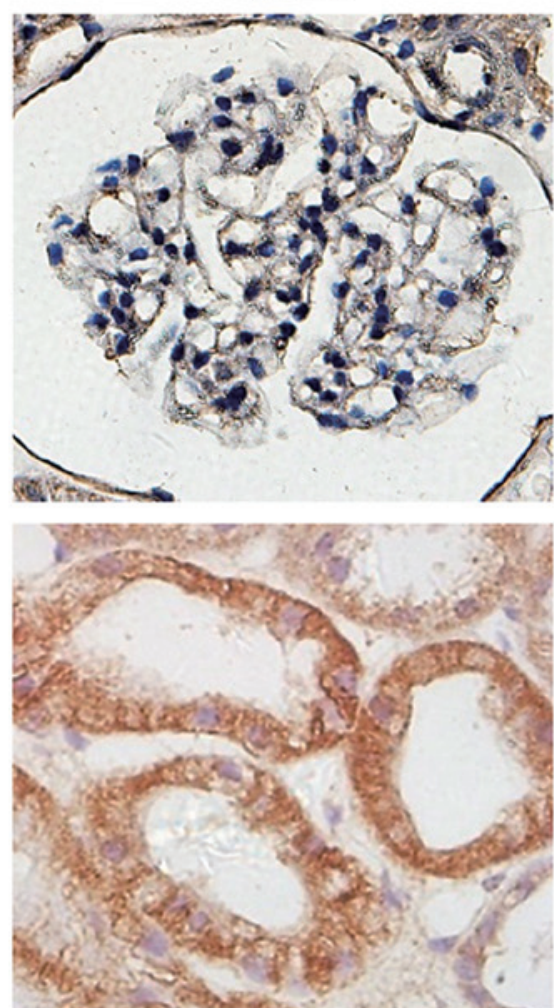

Figure 2. Expression of AQP2 in kidney tissues in the control, NS without edema and NS with edema groups (magnification, $\mathrm{x} 400)$ ). Representative micrographs in each group demonstrate the immunostaining results for AQP2 in renal tubules (bottom panel) and glomeruli (top panel). AQP, aquaporin; NS, nephrotic syndrome. 


\section{Control group}
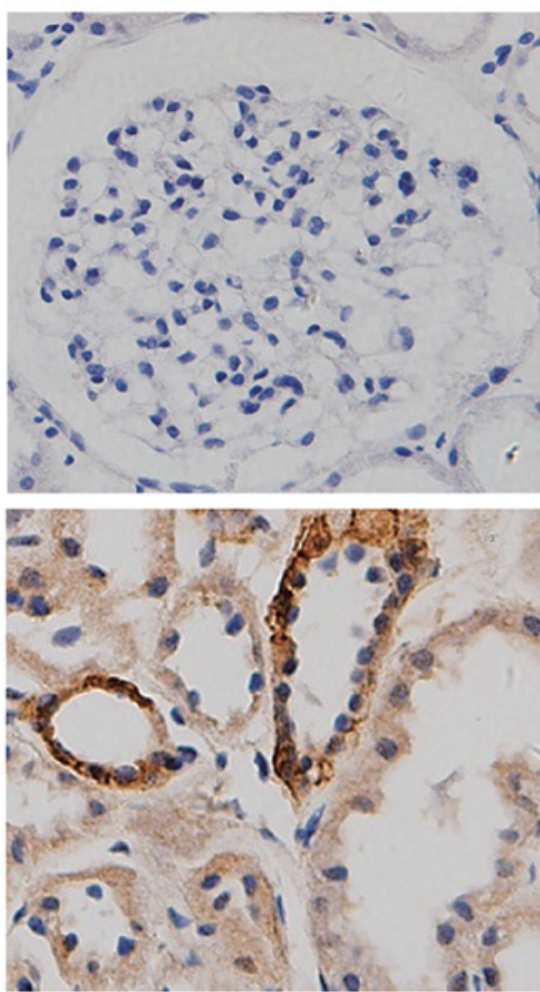

NS without edema
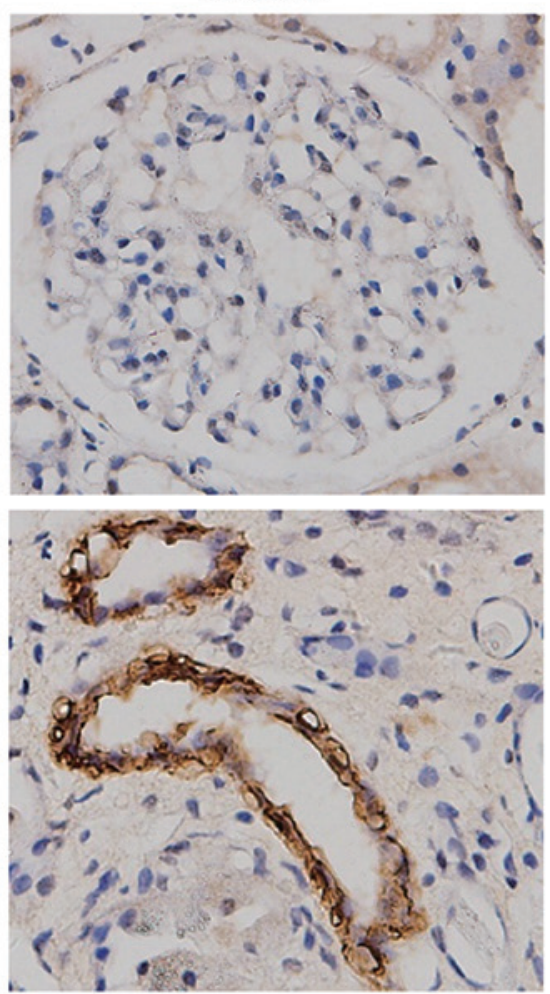

NS with edema
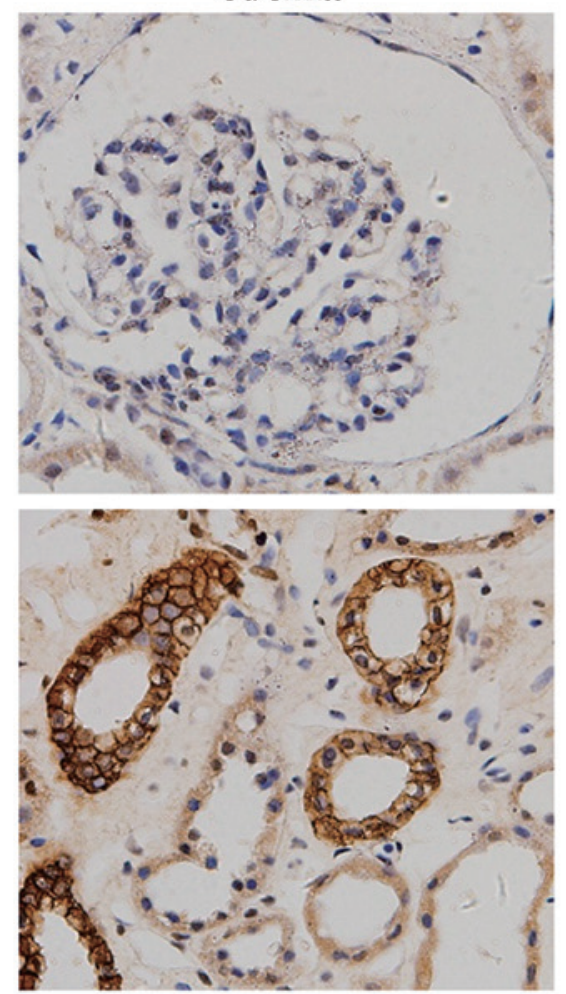

Figure 3. Expression of AQP3 in kidney tissues in the control, NS without edema and NS with edema groups (magnification, $\mathrm{x} 400$ ). Representative micrographs in each group demonstrate the immunostaining results for AQP3 in renal tubules (bottom panel) and glomeruli (top panel). AQP, aquaporin; NS, nephrotic syndrome.

\section{Control group}
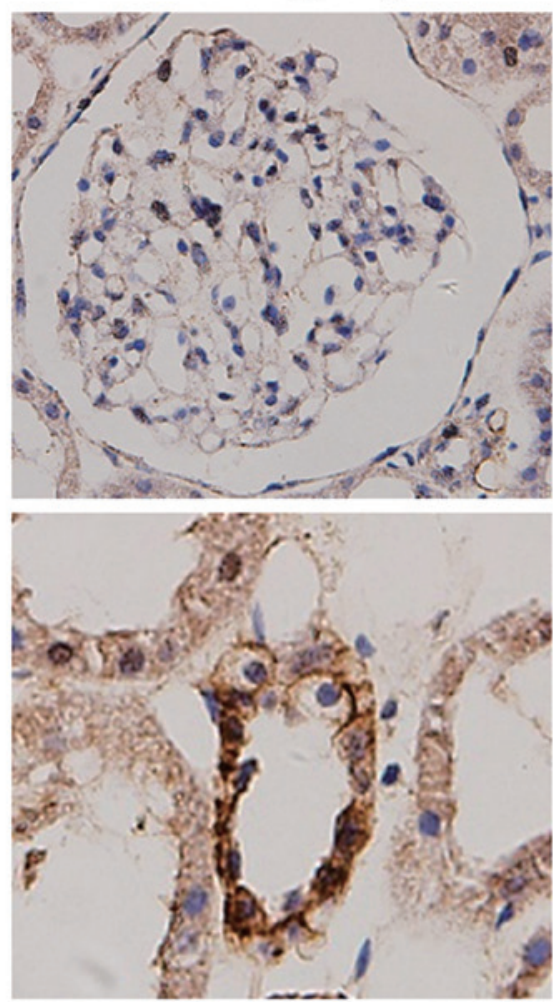

NS without edema
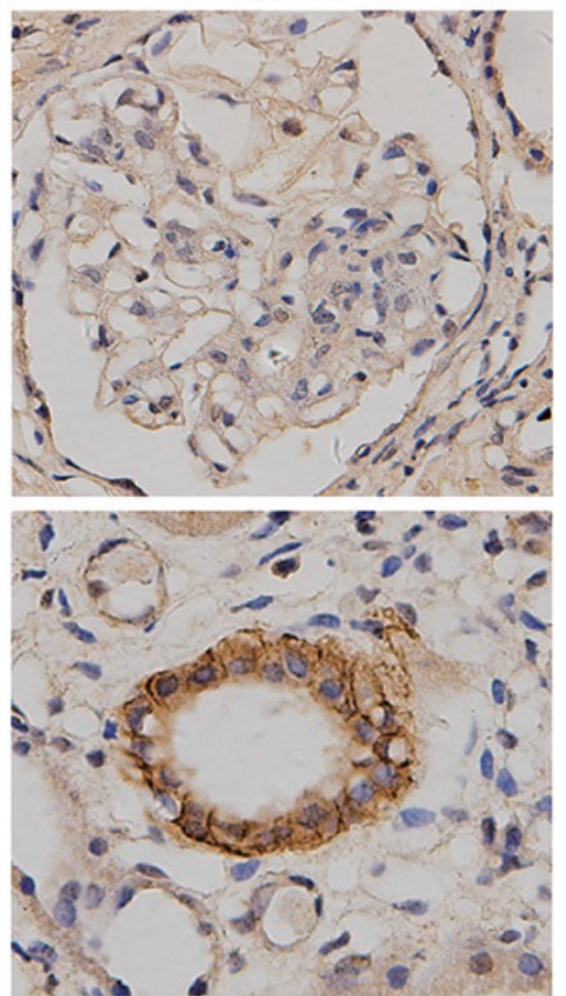

NS with edema
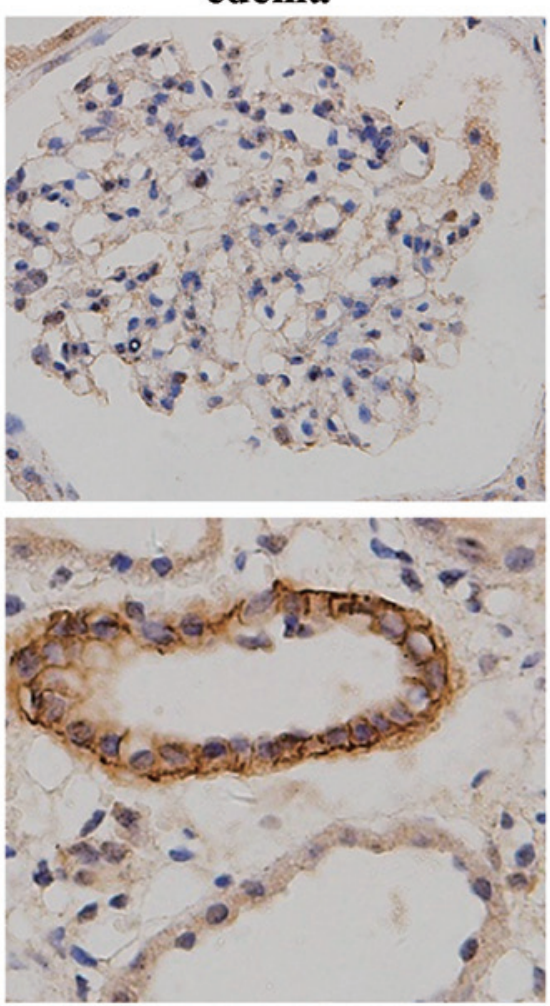

Figure 4. Expression of AQP4 in kidney tissues in the control, NS without edema and NS with edema groups (magnification, $\mathrm{x} 400$ ). Representative micrographs in each group demonstrate the immunostaining results for AQP4 in renal tubules (bottom panel) and glomeruli (top panel). AQP, aquaporin; NS, nephrotic syndrome. 
Table IV. Correlation between AQP expression in kidney tissues and urinary AQP levels in patients with NS.

\begin{tabular}{lcc}
\hline AQP & $\mathrm{R}$ & $\mathrm{P}$-value \\
\hline 1 & 0.1558 & 0.2605 \\
2 & 0.6198 & $<0.001$ \\
\hline
\end{tabular}

AQP, aquaporin; NS, nephrotic syndrome.

of $\mathrm{AQP} 2$ in the urine was not statistically associated with the expression of this protein in kidney tissues in all patients; while, a correlation was observed between these parameters in patients with NS (Table IV).

AQP3 expression in kidney tissues. AQP3 was strongly expressed in the collecting ducts, and was not detected in the glomerulus (Fig. 3). No statistically significant differences were detected among the groups (all P>0.05, Table II).

AQP4 expression in kidney tissues. AQP4 was strongly expressed in the collecting ducts of certain patients, and was not detected in the glomerulus (Fig. 4).

\section{Discussion}

Edema is a key clinical symptom of NS and is considered to be associated with water and sodium retention, in addition to increases in peripheral capillary permeability (3). The mechanism underlying the retention of water and sodium remains unclear. It has been hypothesized to be stimulated by disorders of neurological and humoral factors, resulting from hypovolemia. However, hypovolemia is not present in all patients. Indeed, the majority exhibit normal or higher than average blood volumes (3). Therefore, in recent years, the majority of scholars agree that water and sodium retention are primary features of patients with NS (3).

The present study demonstrated that AQP1 expression was significantly reduced in the proximal tubules of patients with NS with edema. In addition, the quantity of AQP1 in the urine in this group was higher than that in the control group. AQP1, also termed prototype water channel, is a six-transmembrane pore protein, and its tetramers on the cell membrane bidirectionally transport water molecules in and out of the cell (5). AQP1 is expressed in various tissues, including the liver, lung, gallbladder, salivary glands, lacrimal glands, intestine and kidney (14). In the kidney, AQP1 is predominantly expressed in the membranes of the proximal tubular epithelial cells, mediating transmembrane transportation of water and participating in water reabsorption $(3,15)$. AQP1 is responsible for the transportation of $90 \%$ of glomerular filtrates by the proximal tubules $(3,15)$. In addition, transmembrane water flow is regulated by AQP1 levels. A previous study demonstrated that AQP1 knockout mice develop diabetes insipidus, exhibiting a greater than 7-fold increase in water intake and urine output (16). In addition, the urine disorders observed in AQP1 knockout mice resulted from proximal tubule reabsorption loss and medullary hypotonicity, which in turn resulted from the loss of the countercurrent multiplication mechanism (16). In isolated proximal tubule perfusion experiments, transepithelial and permeable water channels were reduced by $80 \%$, suggesting that the key water transport pathway driven by osmotic pressure was AQP1-mediated transepithelial water transportation $(3,15,17)$. In the current study, it was observed that AQP1 expression was reduced in patients with NS with edema, and their urine samples also contained higher quantities of AQP1, although these differences were not statistically significant. This may represent a compensatory response to water and sodium retention in the body, in which AQP1 molecules are excreted into the urine in order to inhibit the reabsorption of water by the kidneys; thus resulting in reduced AQP1 levels in the kidneys, with NS patients with edema exhibiting water diuresis due to a concentrating defect. However, a statistical difference was not observed in the current study. Therefore further investigations with larger sample sizes are required to confirm or refute these observations.

Notably, the expression of AQP2 observed in the kidney, and the quantity of AQP2 in the urine, of the two NS groups was significantly greater than that in the control group, with a greater effect observed in the patients with NS with edema. In addition, it was demonstrated that AQP2 levels in the urine of patients with NS were significantly positively correlated with the AQP2-positive index in kidney tissues. These observations suggest that increased AQP2 expression is associated with the reabsorption of water, which may result in water and sodium retention, and the subsequent development of edema. In addition, the level of AQP2 in the urine may predict the expression of AQP2 in kidney tissues. AQP2 was one of the AQPs in the kidney that were cloned by Fushimi et al (18) in 1993, and is composed of 271 amino acids. Notably, AQP2 was exclusively found on the luminal side of the principal cells and vesicles close to the luminal side of collecting ducts (also termed the collecting duct water channel) (19). As the most widely-distributed and antidiuretic-sensitive AQP subtype in the kidney (20), AQP2 is considered to be the key AQP in principal cells in the epithelium of renal collecting ducts, and the critical protein involved in water reabsorption (4). Therefore, it serves a vital function in the maintenance of water balance and the regulation of body fluid volume and osmotic pressure. The expression of AQP2 is regulated by arginine vasopressin (AVP). Short term regulation is triggered by AVP and transfers AQP2 from the cytoplasm to the luminal membrane vesicles, increasing its density in luminal membranes and thereby increasing their permeability to water (21). As a result, water is taken up by endocytosis, transported to the interstitium by the medullary osmotic gradient and transferred back to the blood stream through renal vessels, in a reversible process that lasts $\sim 3$ min (22). Long-term regulation occurs when AVP levels continue to rise, resulting in an increase in AQP2 mRNA levels, with AQP2 molecules being removed in the urine (23). Previous studies in rats have demonstrated that $3 \%$ of AQP2 molecules produced by kidney tissues is discharged through the urine (24). In addition, the use of diuretics significantly increased urine output and the quantity of AQP2 in urine (25). Furthermore, Zehuang granules have been demonstrated to significantly lower the expression of $\mathrm{AQP} 2$ and the quantity of AQP2 in the urine (26). The current study identified that urine levels of AQP2 were not statistically associated with 
its expression in kidney tissues in all patients; while, these parameters were statistically associated in patients with NS. It is plausible that control patients exhibited very low protein levels.

Furthermore, it was demonstrated that AQP3 expression was higher in patients with edema than in the other two groups, although this results was not statistically significant. AQP3 is permeable to small molecules, such as glycerol and urea, in addition to water. It is expressed in various tissue types, including the trachea, lung, gastrointestinal mucosa and the kidneys (5). AQP3 was demonstrated to be distributed in the basolateral membranes of epithelial cells in the collecting ducts (27). The mechanisms regulating the expression of AQP3 remain unclear. Previous studies have suggested that it is affected by osmotic pressure, while others have hypothesized that AVP levels are involved in the expression of AQP3 (28-30). An association with $\mathrm{pH}$ has also been previously postulated (31), and AQP3 has been shown to be closely associated with the urinary concentrating ability of kidneys, with its loss resulting in polydipsia, polyuria and renal atrophy in mice (32). AQP3 has been reported to produce partially concentrated urine, and following treatment with D-arginine vasopressin or water deprivation, AQP3 knockout mice have been reported to produce partially concentrated urine. (33). Nephrogenic diabetes insipidus in AQP3 knockout mice may be due to the reduced water permeability of the epithelial basement membranes of cortical collecting ducts (33). Another symptom observed in AQP3 knockout mice was functional renal obstruction resulting from severe polyuria (33). The mice exhibited hydronephrosis, renal atrophy and progressive renal failure at 4-6 weeks of age, and died at 2-3 months of age. The current study suggested that AQP3 participates in the regulation of water in the kidneys, but does not serve a vital function. However, due to the limited sample size, further studies with a greater number of participants would be beneficial in confirming these results. It has been demonstrated that collecting duct AQPs in puromycin aminonucleoside (PAN)-nephrotic rats exhibited a reduction of 87 and $70 \%$ in the level of AQP2 and AQP3, respectively, indicating that PAN-nephrosis is associated with an extensive downregulation of collecting duct water channel expression, despite increases in circulating AVP (34).

In the current study, AQP4 was exclusively expressed in the collecting ducts in certain patients, but was not expressed in the glomerulus. AQP4 was distributed predominantly in the inner zone of the outer medulla, outer $1 / 3$ of the inner medulla and the S3 segment of the proximal tubule. AQP4 was observed to participate in water reabsorption and the concentration of urine (35). Additional studies have identified that AQP4 knockout mice exhibit lower levels of water permeability in the medulla and only exhibit slight defects in concentrating urine $(36,37)$. However, the mechanism regulating the expression of AQP4 remains unclear. Cerebral ischemia or infarction models have suggested that oxygen concentrations may have be involved in this process $(38,39)$. In addition, the involvement of osmotic pressure has been suggested (40). It is unclear whether the fact that AQP4 was detected only in certain samples was due to the difference in sections extracted by biopsy, or to the different regulation of water by the kidneys. However, renal biopsy specimens were predominantly obtained from the renal cortex or corticomedullary junctions. Animal experiments are required to further clarify this issue.

In conclusion, by assessing the expression levels of AQPs in kidney tissues, the present study demonstrated that a correlation exists between edema in NS and AQP levels. Of note, the different AQP subtypes did not share the same pattern of expression. Water and sodium retention may be inhibited by compensatory reductions in AQP1 expression in the kidney. AQP2 was highly expressed in the kidney and participated in the reabsorption of water, which may have resulted in water and sodium retention. Whether AQP3 and AQP4 are associated with edema in NS requires further investigation with a larger sample size or the use of animal experiments. The observations of the current study provide a basis for investigating the roles of AQPs in the pathogenesis of NS.

\section{Acknowledgements}

The current study was supported by a grant from the Fudan University Shanghai Medical College Youth Fund (grant no. $11 \mathrm{~L}-8)$.

\section{References}

1. Singhal R and Brimble KS: Thromboembolic complications in the nephrotic syndrome: Pathophysiology and clinical management. Thromb Res 118: 397-407, 2006.

2. Goodman, Boissonnault and Fuller: Implications for the physical therapist. In: Pathology, 2003.

3. Siddall EC and Radhakrishnan $\mathrm{H}$ : The pathophysiology of edema formation in the nephrotic syndrome. Kidney Int 82: 635-642, 2012

4. Verkman AS: Physiological importance of aquaporin water channels. Ann Med 34: 192-200, 2002.

5. Agre P: The aquaporin water channels. Proc Am Thorac Soc 3:5-13, 2006.

6. Verkman AS: Applications of aquaporin inhibitors. Drug News Perspect 14: 412-420, 2001.

7. Nielsen S, Frøkiaer J, Marples D, Kwon TH, Agre P and Knepper MA: Aquaporins in the kidney: From molecules to medicine. Physiol Rev 82: 205-244, 2002.

8. Sprinivasan H: A simple and direct method for simultaneously measuring the angles of adjacent finger joints. Plast Reconstr Surg 57: 524-525, 1976.

9. Krane CM and Goldstein DL: Comparative functional analysis of aquaporins/glyceroporins in mammals and anurans. Mamm Genome 18: 452-462, 2007.

10. Papadopoulos MC, Krishna S and Verkman AS: Aquaporin water channels and brain edema, Mt Sinai J Med 69: 242-248, 2002 .

11. King LS, Yasui $M$ and Agre P: Aquaporins in health and disease. Mol Med Today 6: 60-65, 2000

12. Xu DL, Martin PY, Ohara M, et al: Upregulation of aquaporin-2 water channel expression in chronic heart failure rat. J Clin Invest 99: 1500-1505, 1997.

13. Wright G, Soper R, Brooks HF, et al: Role of aquaporin-4 in the development of brain oedema in liver failure. J Hepatol 53: 91-97, 2010.

14. Mobasheri A and Marples D: Expression of the AQP-1 water channel in normal human tissues: A semiquantitative study using tissue microarray technology. Am J Physiol Cell Physiol 286: C529-C537, 2004.

15. Verkman AS: Roles of aquaporins in kidney revealed by transgenic mice. Semin Nephrol 26: 200-208, 2006.

16. Verkman AS: Renal concentrating and diluting function in deficiency of specific aquaporin genes. Exp Nephrol 10: 235-240, 2002 .

17. Nawata CM, Evans KK, Dantzler WH and Pannabecker TL: Transepithelial water and urea permeabilities of isolated perfused Munich-Wistar rat inner medullary thin limbs of Henle's loop. Am J Phsyiol Renal Physiol 306: F123-F129, 2014. 
18. Fushimi K, Uchida S, Hara Y, Hirata Y, Marumo F and Sasaki S: Cloning and expression of apical membrane water channel of rat kidney collecting tube. Nature 361: 549-552, 1993

19. Okutsu R, Rai T, Kikuchi A, et al: AKAP220 colocalizes with AQP2 in the inner medullary collecting ducts. Kidney Int 74 $1429-1433,2008$

20. Kwon TH, Nielsen J, Masilamani S, et al: Regulation of collecting duct AQP3 expression: Response to mineralocorticoid. Am J Physiol Renal Physiol 283: F1403-F1421, 2002.

21. Moeller HB, Praetorius J, Rützler MR and Fenton RA: Phosphorylation of aquaporin-2 regulates its endocytosis and protein-protein interactions. Proc Natl Acad Sci USA 107: 424-429, 2010.

22. Eto K, Noda Y,Horikawa S, Uchida S and Sasaki S: Phosphorylation of aquaporin-2 regulates its water permeability. J Biol Chem 285 40777-40784, 2010.

23. Camici M: Molecular pathogenetic mechanisms of nephrotic edema: Progress in understanding. Biomed Pharmacother 59: 215-223, 2005.

24. Rai T, Sekine K, Kanno K, et al: Urinary excretion of aquaporin-2 water channel protein in human and rat. J Am Soc Nephrol 8: 1357-1362, 1997.

25. Kwon TH, Nielsen J, Knepper MA, Frøkiaer J and Nielsen S: Angiotensin IIAT1 receptorblockadedecreases vasopressin-induced water reabsorption and AQP2 levels in NaCl-restricted rats. Am J Physiol Renal Physiol 288: F673-F684, 2005.

26. Guo B, Li F and Li X: Regulatory effect of ze-huang granule on AQP2 expression in kidney and urine in diabetic nephropathy rats. Zhong Guo Zhong Xi Yi Jie He Shen Bing Za Zhi 11: 121-124, 2010 (In Chinese).

27. Ishibashi K, Sasaki S, Fushimi K, Yamamoto T, Kuwahara M and Marumo F: Immunolocalization and effect of dehydration on AQP3, a basolateral water channel of kidney collecting ducts. Am J Physiol 272: F235-F241, 1997.

28. Nejsum LN, Kwon TH, Marples D, et al: Compensatory increase in AQP2, p-AQP2 and AQP3 expression in rats with diabetes mellitus. Am J Physiol Renal Physiol 280: F715-F726, 2001.

29. García-Delgado M, Peral MJ, García-Benítez O, Carreras O and Ilundáin AA: Prolonged ethanol ingestion increases renal AQP2 and AQP3 expression in adult rats and in their offspring. J Membr Biol 198: 89-94, 2004.
30. Gong H, Wang K, Kwon TH, Jonassen T, Frøkiaer J and Nielsen S: Reduced renal expression of AQP2, p-AQP2 and AQP3 in haemorrhagic shock-induced acute renal failure. Nephrol Dial Transplant 18: 2551-2559, 2003.

31. Zeuthen T and Klaerke DA: Transport of water and glycerol in aquaporin 3 is gated by $\mathrm{H}(+)$. J Biol Chem 274: 21631-21636, 1999.

32. Yang B, Ma T and Verkman AS: Erythrocyte water permeability and renal function in double knockout mice lacking aquaporin-1 and aquaporin-3. J Biol Chem 276: 624-628, 2001.

33. Ma T, Song Y, Yang B, et al: Nephrogenic diabetes insipidus in mice lacking aquaporin-3 water channels. Proc Natl Acad Sci USA 97: 4386-4391, 2000.

34. Apostol E, Ecelbarger CA, Terris J, Bradford AD, Andrews $\mathrm{P}$, and Knepper MA: Reduced renal medullary water channel expression in puromycin aminonucleoside - induced nephrotic syndrome. J Am Soc Nephrol 8: 15-24, 1997.

35. van Hoek AN, Ma T, Yang B, Verkman AS and Brown D Aquaporin-4 is expressed in basolateral membranes of proximal tubule S3 segments in mouse kidney. Am J Phsyiol Renal Physiol 278: F310-F316, 2000.

36. Terris J, Ecelbarger CA, Marples D, Knepper MA and Nielsen S: Distribution of aquaporin-4 water channel expression within rat kidney. Am J Physiol 269: F775-F785, 1995.

37. Wang JF, Wang ZY, Wu N, Yan HT and Li J: Effects of aquaporin 4 deficiency on opioid receptors characteristics in naive and chronic morphine-treated mice. Neurosci Lett 457: 111-114, 2009.

38. Yamamoto N, Yoneda K, Asai K, et al: Alterations in the expression of the AQP family in cultured rat astrocytes during hypoxia and reoxygenation. Brain Res Mol Brain Res 90: 26-38, 2001.

39. Aoki K, Uchihara T, Tsuchiya K, Nakamura A, Ikeda K and Wakayama Y: Enhanced expression of aquaporin 4 in human brain with infarction. Acta Neuropathol 106: 121-124, 2003.

40. Hashido M, Kidera A and Ikeguchi M: Water transport in aquaporins: Osmotic permeability matrix analysis of molecular dynamics simulations. Biophys J 93: 373-385, 2007. 